\title{
Endoplasmic Reticulum Aminopeptidase-1 (ERAP-1) Mutations in People with Familial Mediterranean Fever (FMF) Gene Mutations Without Disease
}

\section{Resat Dabak, Gulbuz Sezgin, Faith Oner Kaya, Feride Sinem Akgun, Oya Bayramicli and Selim Nalbant*}

Department of Internal Medicine, Maltepe University, Turkey

*Corresponding Author: Selim Nalbant, Department of Internal Medicine, Maltepe University, Turkey.
Received: October 22, 2020

Published: November 21, 2020

(C) All rights are reserved by Selim Nalbant.

\begin{abstract}
Background and Study Aim: To date, especially alterations of genes on exon 10 have been considered in Mediterranean fever (MEFV), but it is not clear whether all these alterations are disease-causing mutations.

We studied ERAP-1 gene mutations in people with FMF gene mutations without FMF phenotype. We compared our results with our previous study which we studied ERAP-1 gene mutations in patients with FMF and with ulcerative colitis (which has been shown to have a relation with ERAP-1 gene mutations before).

Patients and Methods: This is a retrospective study. We studied ERAP-1 gene mutation in blood samples (had been kept in $-70^{\circ} \mathrm{C}$ before for different studies) from 51 people with FMF gene mutations without FMF disease. Some of these people also were followed for about 5 years whether FMF comes out. .

Results: There were 10 cases without ERAP-1 mutations at Exon-3 and 6 cases without ERAP-1 mutations at Exon-10. Any ERAP1 mutations were not obtained in three cases both for Exon-3 and 10.

There were 41 ERAP-1 gene mutations at Exon-3 and 48 at Exon-10. Exon-3 mutations were usually Codon 127 mutation for 35 patients whether it was single mutation or with multiple mutations. It was totally different our ERAP-1 mutation in patients with FMF for Exon-3. Because in our previous study, if there were ERAP-1 gene mutations at exon-3, [c.380G>C p.(Arg127Pro)] mutation always existed in patients with FMF.

Conclusion: As a conclusion, ERAP-1 may be the second needed genetic mutation to produce the disease, 2) Certain ERAP-1 genetic mutation may produce FMF together with certain pyrin gene mutation.
\end{abstract}

Keywords: Familial Mediterranean Fever; ERAP-1; Inflammation; M694

\section{Introduction}

Familial Mediterranean Fever (FMF) is an autosomal recessive and accepted as a monogenic disorder that frequently affects Turkish, North Africans, Jewish, Arabic, and Armenian populations [1]. It is characterized by episodic abdominal pain, arthritis, pericarditis, pleurisy, fever and skin rash. However, effort of explaining the pathophysiology of the disease provide to find the genetical back- ground of the FMF. Finding the pyrin mutation, made a new line of vision to not only to FMF but all the autoinflammatory diseases [1-13].

However, we presumed that single mutations in pyrin gene is not enough to produce FMF phenotype. Because we believe that to accept this disease as monogenic with its heterogeneous clinical aspects may be contradictory. There may be some additional 
Endoplasmic Reticulum Aminopeptidase-1 (ERAP-1) Mutations in People with Familial Mediterranean Fever (FMF) Gene Mutations Without Disease

mutations for the manifestations of FMF disease out of pyrin gene mutations. In our previous study we almost had showed that there are more ERAP-1 mutations (especially in exon10) in patients with FMF in accordance with patients in ulcerative colitis group which had been shown the relation before [1]. In the light of these results, we concluded that there may be a strong susceptibility with ERAP-1 gene mutations for occurring clinics of FMF. All patients with FMF had FMF mutations (mostly M694) in our previous study. Therefore, our FMF population was quite homogeneous.

The ERAP1 gene provides instructions for making a protein called endoplasmic reticulum aminopeptidase. Basically, it regulates aminopeptidase which is an enzyme that cleaves other proteins into small peptides to provide the recognizing the antigenic determinants. If the immune system recognizes these peptides as foreign (such as viral or bacterial peptides) it responds by triggering the infected cell to self-destruction [12-17].

Therefore, in this current study we studied ERAP-1gene mutations in people with $F M F$ gene mutations but without FMF disease (without phenotype). We tried to verify that manifest FMF disease needs additional mutation(s) by showing people with $M E F V$ gene mutations but without disease. We also compared our results with our previous study which we studied ERAP-1 gene mutations (which is possible additive effect on $M E F V$ gene mutations) in patients with FMF and with ulcerative colitis (which has been shown to have a relation with ERAP-1 gene mutations before).

\section{Materials and Methods}

This is a kind of retrospective study. We studied ERAP-1gene mutation in blood samples (which had been kept in $-70^{\circ} \mathrm{C}$ before for some previous studies) from 51 people with $F M F$ gene mutations without FMF disease. They invite again to our center, if they had any attacks of the disease during the out of control of us. If the cases with $M E F V$ gene mutations had any FMF attacks during this uncontrolled period was not included to the normal phenotype group. Some of these people also had been followed for about 5 years but any FMF clinics has not been happened.

The mean age of the cases was $38.90 \pm 7.09$ (M/F: 15/36) years.
Helsinki and local approval was received from the Ethics Committee of Training Hospital of Maltepe Medical Faculty Maltepe -İstanbul. Informed consent was obtained from all patients.

\section{Detection of serum ERAP-1gen mutations}

Deposited serums in Eppendorf tubes at $-70^{\circ} \mathrm{C}$ were used for ERAP-1 mutations. Analysis of ERAP-1 gene mutations at exon-3 and exon-10 were done with PCR-DNA folders. Primer pairs were used at 0.6 pico mole $/ \mathrm{mL}$. The other PCR components were 10 $\mathrm{mM}$ Tris- $\mathrm{HCl}\left(25^{\circ} \mathrm{C} \mathrm{pH}: 8.8\right), 50 \mathrm{mM} \mathrm{KCl}, 0.2 \mathrm{mM}$ deoksi-nukleotit3-phosphate [dATG, dGTP, dCTP, dTTP (Fermentas, Lithuania)] and $15 \mathrm{mM} \mathrm{MgCl}$. ERAP-1 gene primers at exon-3: 5'AGT TCA ACA GCA AAG GGA ATT3', 5'TTT TGC TTT TGT ACA TTT G3' ERAP-1 gene primers at exon-10: 5'CTC CTC AGA GGG ATT AAC ATA, 5'TTA ACA GTG TTC CTG CAG TTG CG3'. After denaturation and hybridization (Applied Biosystems, USA) of PCR-products, amplification of right gene region was checked by agarose gel.

After purification of samples (Bio Basic, Canada), a new PCRcycling was done and purified again. At the end of this process, samples were loaded to the DNA folders analyzer (Applied Biosystems $3130 x l$, USA). Obtained results were analyzed by SeqScape ${ }^{\circledR}$ Software v3.0.

Results were given as both first cDNA and and second protein changes.

\section{Statistical analysis}

Statistical analysis was done by SSPS statistical software (SPSS for windows 10.0, Inc., Chicago, IL, USA). Groups were compared with Marginal Homogeneity. Statistical significance was defined as $\mathrm{p}<0.05$.

\section{Results}

The results are shown in figure 1 . There were 34 cases with single mutation (18 cases with M694V mutation, 5 cases with E148Q 4 cases with M680I, 4 cases with V726A, 3 cases with A744S) and 17 cases with multiple mutations ( 5 cases with M694V and E148Q 1 case with M694V and 680I, 4 cases with M694V and V726A, 2 cases with M680I and V726A, 4 cases with E148Q and P369S, 1 cases with M680I and E148Q). 


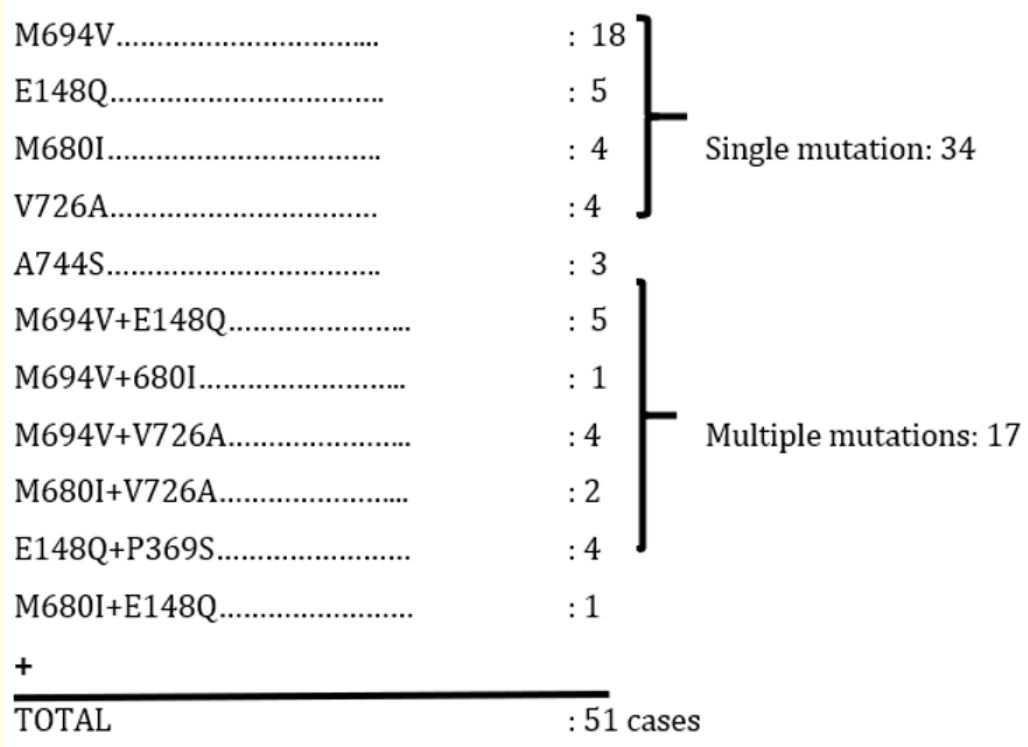

Figure 1: The distribution of FMF gene mutations.

There were 11 cases without ERAP-1 mutations at Exon-3, 6 cases without ERAP-1 mutations at Exon-10. ERAP-1 mutations were not obtained in three cases both for Exon-3 and 10 (Table 1).

There were 40 cases with ERAP-1 gene mutations at Exon-3 and 45 cases with ERAP-1 gene mutations at Exon-10. Exon-3 mutations were usually c.380 G>C p.(Arg127Pro) mutation for 35 patients whether it was single mutation or with multiple mutations (33 single mutations and 2 multiple mutations) (Table 1). It was totally very similar with our previous study showing ERAP-1 mutation in patients with FMF for Exon-3 (Table 2).

There were 45 single ERAP-1 gene mutations at exon-10. [c.1359T >C p.(Ser127Pro)] was the only mutation type for all cases whether it was heterozygote or homozygote. It was also totally different for our previous study in patients with FMF who had single mutation [c.1359T >C p.(Ser453Pro) ] in 10 patients and [c.1359T >C
p.(Ser127Pro)] in three patients FMF showed [c.380G>C p.(Arg127 Pro)]single gene mutation at exon-10 (Table 2).

Dominant mutation for ERAP-1 gene [c.380G>C p.(Arg127 Pro)] at Exon-3 in patient group was significantly similar $(\mathrm{p}<0.005)$ with non-patient group in contradistinction to being at Exon-10. Dominant mutation at Exon-10 was [c.380G >C p.(Ser 453 Ser)] in patient group. This mutation was significantly different between patient group and non-patient group ( $\mathrm{p}>0.7$ ) at Exon-10.

\section{Discussion and Conclusion}

To show the genetic background of FMF, it is very important to understand main mechanism of all this kind of auto-inflammatory disease. In previous studies, it was studied to show correlation of one kind of mutation with FMF. Most of the studies had shown that different kinds of Exon-10 mutations were significantly high in different ethnic groups. However, these findings could not be tested. 
Endoplasmic Reticulum Aminopeptidase-1 (ERAP-1) Mutations in People with Familial Mediterranean Fever (FMF) Gene Mutations Without Disease

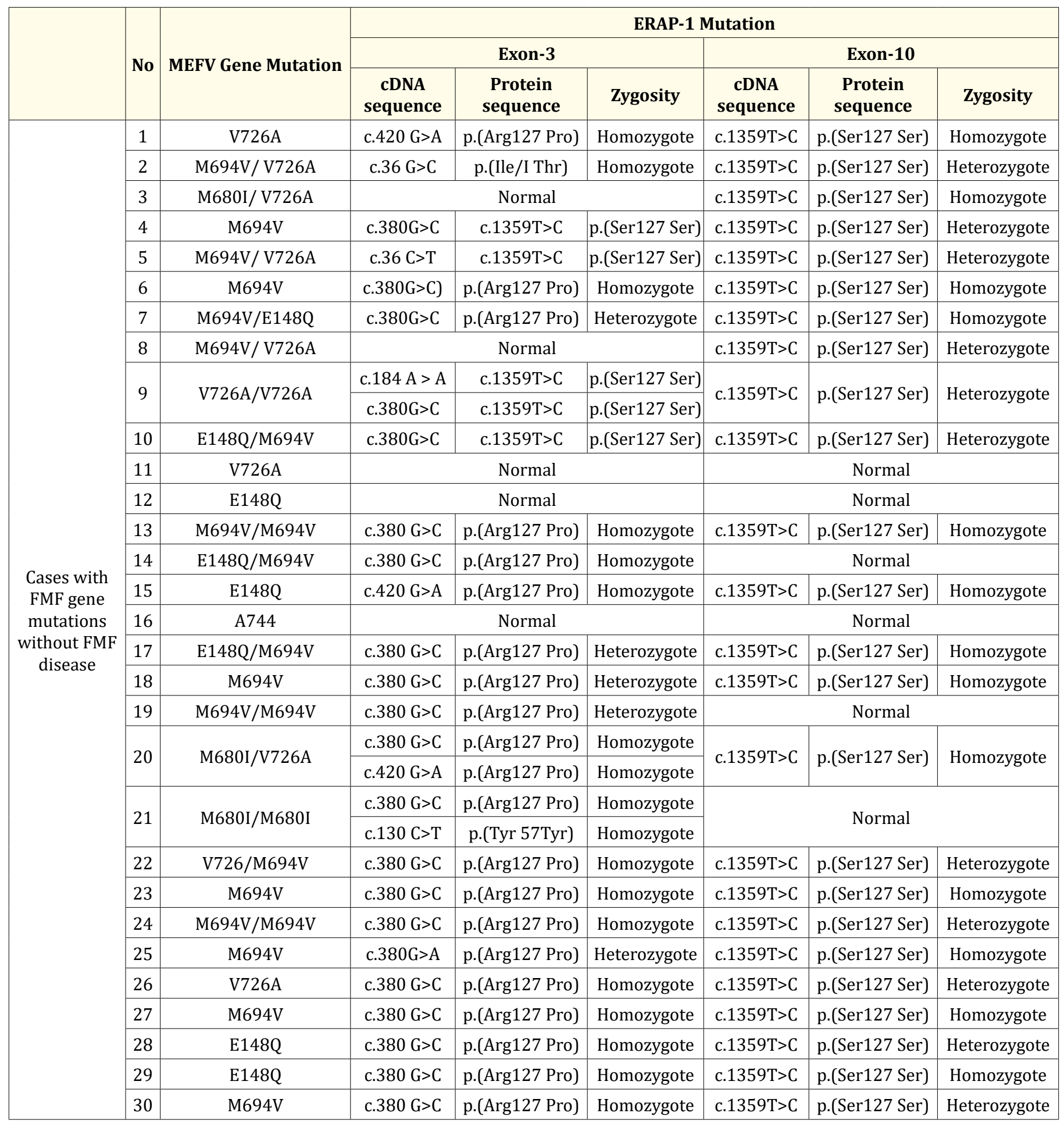


Endoplasmic Reticulum Aminopeptidase-1 (ERAP-1) Mutations in People with Familial Mediterranean Fever (FMF) Gene Mutations Without Disease

08

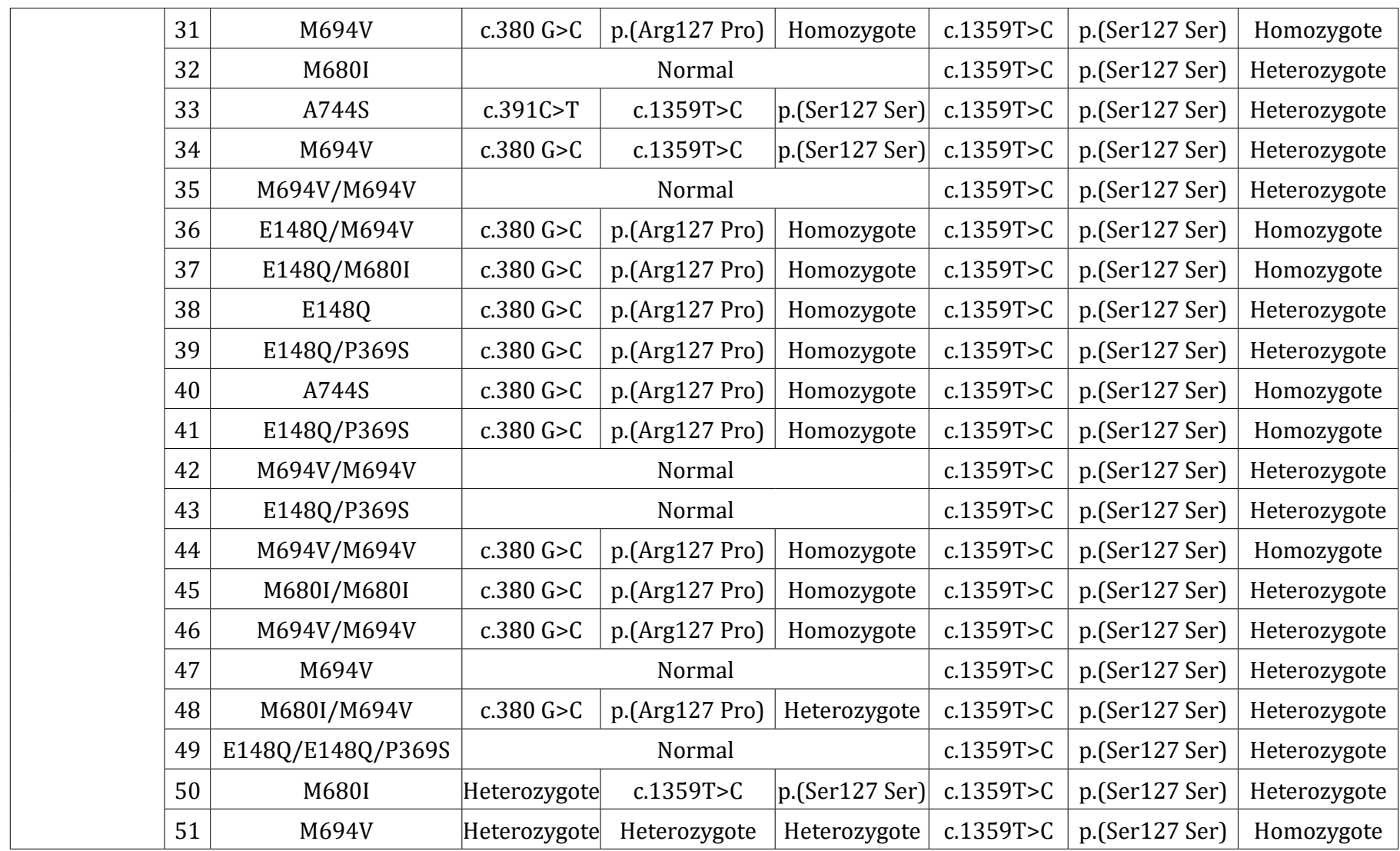

Table 1: Whole documents of the study for patients with FMF mutation without FMF disease.

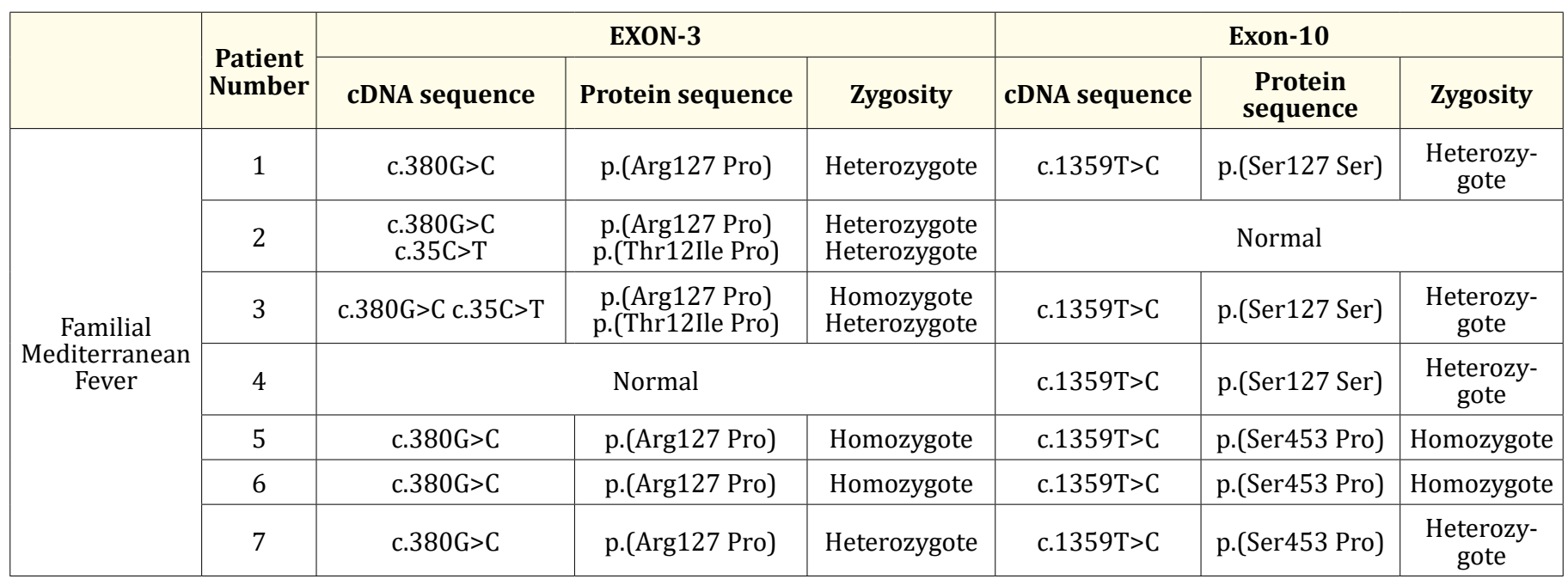


Endoplasmic Reticulum Aminopeptidase-1 (ERAP-1) Mutations in People with Familial Mediterranean Fever (FMF) Gene Mutations Without Disease

09

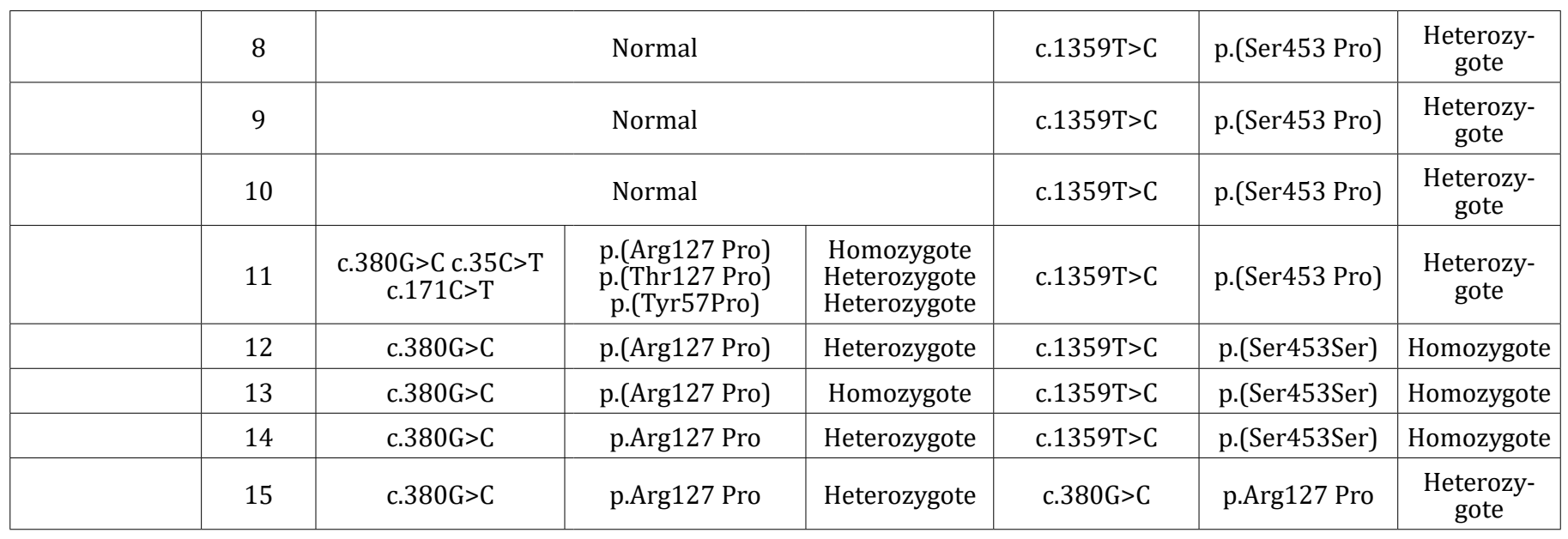

Table 2: Results of ERAP-1 gene mutations in patients with FMF*.

*All of the patient.

Because there is no study done on cases with FMF gene mutation without FMF. On the other hand, FMF can be a polygenic disease according to our thinking of origin.

In this study and in our previous study, for the first time, we showed that if a definite ERAP-1 and classical pyrin gene mutations are together then the possibility of FMF phenotype increases.

In our previous study [c.1359T>C p.(Ser453Pro)] mutation at Exon-10 of ERAP-1 was more common in patient group with FMF. However, in our current study [c.1359T>C p.(Ser127Ser)] is more common in cases with FMF mutations without disease at Exon-10 for ERAP-1. It is [c.380G $>$ C p.(Arg127 Pro)] for cases with FMF and [p.Arg127 Pro (c.380G>C)] for cases without disease at Exon-3. Difference between two group (with cases FMF and without FMF) for ERAP-1mutation is more definite at Exon-10 than Exon-3.

We strongly believe that these results are very important. Because this study may show that 1-) ERAP-1 may be the second needed genetic mutation to produce the disease, 2-) Certain ERAP1 genetic mutation may produce FMF together with certain pyrin gene mutation.

FMF is a very heterogeneous disease which was shown to have pyrin gene mutation before. So, there may be also some other mu- tations together in the same patients. ERAP-1 gene controls a very important enzyme which helps to recognize the proteins. This step is a key step for the initiation of the inflammation. On the other hand, pyrin controls to end the inflammation. The discovery of ERAP mutations in patients with FMF is not an unexpected finding. We believe that we showed the importance of having these two mutations at the same time to have clinically manifestation of FMF as a first time.

We believe that the evaluation of ERAP mutations in FMF patients with further studies will help us to find new tools for the correct diagnosis and current explanation the etiopathology of the disease. This study may help to establish a step to do this for further studies.

\section{Acknowledgement}

We appreciate TAHEV (Turkish Foundation for Family Physicians) for their financial support and also Department of Biology of Cerrahpasa Medical Faculty on the behalf of Prof. Dr. Nur BUYRU who studied these samples for us.

All procedures performed in studies involving human participants were in accordance with the ethical standards of the institu- 
tional and/or national research committee and with the 1964 Helsinki declaration and its later amendments or comparable ethical standards.

\section{Bibliography}

1. Aksentijevich I and Kastner DL. "Genetics of monogenic autoinflammatory diseases: past successes, future challenges". Nature Reviews Rheumatology 7 (2011): 469-478.

2. Gulbuz Sezgin., et al. "The association of endoplasmic reticulum aminopeptidase-1 (ERAP-1) with Familial Mediterranean Fever (FMF)". United European Gastroenterology Journal 4.1 (2016): 92-96.

3. Daniels M., et al. "Familial mediterranean fever: high gene frequency among the non-askenazi jewish populations in Israel". American Journal of Medical Genetics 55 (1995): 311-314.

4. Odabas AR., et al. "Familial mediterranean fever". South Medical Journal 95.12 (2002): 1400-1403.

5. Gershoni-Baruch R., et al. "Male sex coupled with articular manifestations cause a 4-fold increase in susceptibility to amyloidosis in patients with familial mediterranean fever homozygous for the M694V- MEFV mutation". Journal of Rheumatology 30.2 (2003): 308-312.

6. Zaks N., et al. "Analysis of the three most common MEFV mutations in 412 patients with familial mediterranean fever". Israel Medical Association Journal (IMAJ) 5.8 (2003): 585-588.

7. Brik R., et al. "Familial mediterranean fever: clinical and genetic characterization in a mixed pediatric population of jewish and arap patiens". Pediatrics 103.5 (1999): e70.

8. Konstantopoulos K., et al. "Familial mediterranean fever associated pyrin mutations in Greece". Annals of the Rheumatic Diseases 62.5 (2003): 479-481.

9. Medlej HM., et al. "Genetic screening of fourteen mutations in jordanian familial mediterranean fever patients". Human $\mathrm{Mu}$ tation 15.4 (2000): 384.

10. Tunca M., et al. “On behalf of the Turkish FMF Study Group. The results of a nationwide, multicenter analysis of the clinical and genetic characteristics of the Turkish FMF patients (abstract)". Clinical and Experimental Rheumatology 20 (2002): S92.
11. Livneh A., et al. "Criteria for the diagnosis of familial mediterranean fever”. Arthritis Rheum 40.10 (1997): 1879-1885.

12. Dig Giaglis S., et al. "Increased frequency of mutations in the gene responsible for familial Mediterranean fever (MEFV) in a cohort of patients with ulcerative colitis: evidence for a potential disease-modifying effect?" Digestive Diseases 51.4 (2006): 687-692.

13. Fierabracci A., et al. "The putative role of endoplasmic reticulum aminopeptidases in autoimmunity: Insights from genomic-wide association studies". Autoimmune Review (2012).

14. Saric T., et al. "An IFN-gamma-induced aminopeptidase in the ER, ERAP1, trims precursors to MHC class I-presented peptides". Nature Immunology 3 (2002): 1169-1176.

15. Serwold T., et al. "ERAAP customizes peptides for MHC class I molecules in the endoplasmic reticulum". Nature 419 (2002): 480-483.

16. Tanioka T., et al. "Human leukocyte-derived arginine aminopeptidase. The third member of the oxytocinase subfamily of aminopeptidases". Journal of Biological Chemistry 278 (2003): 32275-32283.

17. York IA., et al. "The ER aminopeptidase ERAP1 enhances or limits antigen presentation by trimming epitopes to 8-9 residues". Nature Immunology 3 (2002): 1177-1184.

18. Saveanu L., et al. "Concerted peptide trimming by human ERAP1and ERAP2 aminopeptidase complexes in the endoplasmic reticulum". Nature Immunology 6 (2005): 689-697.

\section{Assets from publication with us}

- Prompt Acknowledgement after receiving the article

- Thorough Double blinded peer review

- Rapid Publication

- Issue of Publication Certificate

- High visibility of your Published work

Website: $\underline{w w w . a c t a s c i e n t i f i c . c o m / ~}$

Submit Article: www.actascientific.com/submission.php

Email us: editor@actascientific.com

Contact us: +919182824667 